\title{
When should I suspect childhood leukaemia?
}

\author{
Philip Connor
}

The paper by Clarke et $a l^{1}$ sheds light upon the initial presenting features of childhood leukaemia. It is a systematic review and meta-analysis of the clinical features present in children with leukaemia at presentation.

The clinical relevance of this is that there are several diseases that are notorious mimics. 'Know syphilis in all its manifestations and relations, and all other things clinical will be added to you'2 stated Osler in a previous century and something similar could be said of leukaemia by contemporary paediatricians. It is the ability of the various leukaemias to mimic prosaic viral infections in some patients, while in others is the stuff of the membership exam 'grey case' that fuels clinicians' concerns about missing the diagnosis. There is no doubt that it is a serious disorder, but it is also relatively 'common' with about 500 children a year being diagnosed in the UK per year. It is likely that a generalist in a regional hospital will come across a few cases each year and be confronted with difficult cases at some point in their day-to-day practice.

In the paper, the authors whittle away at 95 presenting signs and symptoms to find five features present in more than $50 \%$ of children, namely: hepatomegaly (64\%), splenomegaly (61\%), pallor (54\%), fever (53\%) and bruising (52\%). Additionally, there were eight features present in one-third to half of children: recurrent infections (49\%), fatigue (46\%), limb pain (43\%) hepatosplenomegaly (42\%), bruising and petechiae (42\%) lymphadenopathy (41\%), bleeding tendency (38\%) and rash (35\%). The authors conclude that children with unexplained illness require a thorough history and clinical examination

Correspondence to Dr Philip Connor, Department of Paediatric Haematology/Oncology, Noah's Ark Children's Hospital for Wales, Cardiff CF14 4XW, UK; Philip.Connor@wales.nhs.uk that should include palpation of the abdomen, a search for lymphadenopathy and careful inspection of the skin. As a jobbing paediatric haematologist the features found in the paper are high in my mental checklist and my experience in practice mirrors the findings of the paper. This will be useful to the non-specialist clinician at the coalface and will help inform guideline and protocol writers.

Whereas the syphilis that Osler and his contemporaries battled is caused by a single species of infectious agent, the leukaemias of childhood are a number of different diseases adding to the spectrum of their clinical manifestation. Each often requires a specific diagnosis to guide therapy. That therapy is not necessarily always chemotherapy. It has been one of the major paradigm shifts that has happened over recent years how much the management of chronic myelogenous leukaemia has changed. The introduction of tyrosine kinase inhibitors has transformed care for the majority of patients. It is likely that the next several decades will usher in other treatments into the management of other leukaemias that occur in children, as is already happening in the realm of adult haematology, where novel small molecule inhibitors and biological agents are abounding. The specific diagnostic entities are likely to alter, as the new technologies of nextgeneration sequencing and other techniques change our understanding of tumour biology and how to classify them. However, many of these aspects will be in the area of specialist care and may be of little help to the generalist who has an unwell child in front of them.

I am conscious of how lucky I am in a tertiary referral centre to benefit from the hard work of my colleagues who have usually figured out the diagnosis long before I see the patient or transferred to the haematology/oncology ward. The full blood count remains a very good tissue biopsy for leukaemia. Blasts are often present in the blood and clonal populations are usually picked out by flow cytometry, if they are present. Their presence or not is an important caveat that I will elaborate on later. The common leukaemias in children (ALL and AML) often have blasts in the blood, which can be seen on microscopy of the blood film and detected as an aberrant population. The complete diagnostic classification requires the detection of any of the known cytogenetic or molecular events. These are often easier to perform on marrow-derived cells and it is for this reason (and to obtain adequate minimal residual disease assessment samples) that bone marrow aspirates are done. In short the initial diagnosis of 'it is leukaemia' is usually easily made on peripheral blood. The final specific diagnostic entity is a composite based on: clinical features, morphology, flow cytometry and molecular/cytogenetic features.

Some patients who present with an acute leukaemia (ALL and AML) have a different biology with very few, or no detectable, blasts in the blood. These often do not map to any specific molecular or immunophenotypic variant. These are more challenging and require biopsy of the specific tissue. In my experience these tend to be in two different groups. In one, the blasts are stuck in the marrow. The children commonly present as bone pain/rheumatology symptoms. They may or may not have cytopenias initially although they usually develop over time. All becomes clear once the bone marrow aspirate is done. In the second, the blasts have proliferated in an organ in the body. They may be completely absent from the bone marrow and blood. The most common are T-cell blasts that have infiltrated the thymus, but I have had other patients who had B cells (or myeloid cells) infiltrating kidneys or gut or skin. The specific organ requires biopsying if nothing is present in the marrow. Both of these groups are difficult in that there is uncertainty about the diagnosis until the tissue sample is taken and processed. As an example, the group of patients with skin infiltrating ALL tend to have a very indolent course, one child I recall having a rash for nearly a year before it was apparent that it was ALL that was confined to the skin. 
Death in childhood is fortunately a relatively rare occurrence in the developed world, but in the UK overall childhood mortality rate is higher than in some other European countries. ${ }^{3}$ Injury remains the most frequent cause of death after the first year of life, but cancer is the most common medical cause and leukaemia is the most common cancer. Fortunately, leukaemia relapse is now a rare disorder and the most likely outcome for most patients is cure with survival, but early detection and prompt treatment can only be good for patients. Grey cases will still appear from time to time to test all of us, the paper by
Clarke et al adds detail to the presenting features of leukaemia in children.

Competing interests None declared.

Provenance and peer review Commissioned; internally peer reviewed.

To cite Connor P. Arch Dis Child 2016;101:874-875.

Received 22 May 2015

Accepted 31 May 2015

\section{SLinked}

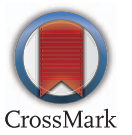

- http://dx.doi.org/10.1136/archdischild-2016-311251
Arch Dis Child 2016;101:874-875.

doi:10.1136/archdischild-2015-308731

\section{REFERENCES}

1 Clarke RT, Van den Bruel A, Bankhead C, et al. Clinical presentation of childhood leaukaemia: a systematic review and meta-analysis. Arch Dis Child 2016;101:894-901.

2 Bean RB, Bean WB, eds. Sir William Osler, aphorisms from his bedside teachings and writings. No 133. Springfield, IL: Charles C. Thomas, 1961.

3 Why children die: death in infants, children, and young people in the UK, RCPCH/NCB/British Association of Child and Adolescent Public Health, May 2014. 